\title{
A Prospective Study of Poisoning Severity Score among Acute Antipsychotic Poisoned Cases Admitted to Menoufia Poison Control Center (MPCC) Throughout One Year
}

\author{
Samy Abd El-Hady Hammad, Naira Fahmi Girgis, Safaa Abd Elzaher Amin, \\ Azza Wagih Zanaty, and Haidy Moustafa Abou Hatab ${ }^{1}$
}

${ }^{1}$ Forensic Medicine and Clinical Toxicology Department, Faculty of Medicine, Menoufia University, Menoufia, Egypt

\begin{abstract}
Introduction: Antipsychotics rank in the top five substance classes involved in human exposures. Overdose of antipsychotic medications is common.

Aim of the work: to study antipsychotic drug poisoning cases that were admitted to MPCC during one year as regarding: socio demographic pattern, their clinical manifestation, investigation and outcome according to poisoning severity score (PSS). Patients and Method: Patients complaining of antipsychotic drug overdose admitted to MPCC from $1^{\text {st }}$ October 2012 to $30^{\text {th }}$ September 2013 were included. The socio-demographic and clinical data were collected from each patient in a designed clinical toxicological sheet. The studied cases were classified according to poisoning severity score. Results: The total patient's number was 60 patients. Females outnumbered males. Cases $33.3 \%$ below 10 years old were males, while females above 40 years old constituted $50.2 \%$. Accidental poisoning was in males below age of 10 years from rural side, on the other hand $75 \%$ of females exposed to suicidal mode mainly from urban area. Regarding (PSS); most of cases were of moderate degree and the least were of severe and fatal degrees. Palpitation and abnormal movements were the commonest symptoms. Sinus tachycardia was the most prominent ECG changes seen in $20 \%$ of cases. ICU admission was indicated for $16.7 \%$ of cases. Improvement has occurred in $40 \%$ of cases, while only one case died. Conclusion and Recommendation: Significant relation was found between PSS and poison history as regard Co-ingestion and patients on antipsychotic therapy. Cases with moderate severity toxicity had palpation and abnormal movements. Acute antipsychotic overdose seldom results in death. Therefore it is recommended to improve awareness of physician about appropriate pharmacologic treatment of psychiatric problems.
\end{abstract}

Keywords Antipsychotics - toxicity- poisoning severity score.

\section{Introduction}

$\mathrm{M}$ ajor tranquilizer usually refers to antipsychotics (Ananth et al., 2004). Antipsychotics can abort specific psychotic symptoms without impairing intellectual ability and level of consciousness. In addition, they are referred to as neuroleptics because of the effective changes they induce, including decrease aggressive behavior and emotional liability (Kwiatkowsk, 2008).

Antipsychotic drugs were classified into first "typical" antipsychotics and second "Atypical" antipsychotics. The first-generation neuroleptic agents, also known as major tranquilizers, comprise a group of several classes of drugs (Butyrophenones, Dibenzoxazepines, Dihydroindolone, Diphenyl butylpiperidine, Phenothiazines, Thioxanthenes) (Haddad and Dursun, 2008).

Second "Atypical" antipsychotics the newer second-generation include Benzepines, Olanzapine, Quetiapienethe. (Shirzadi and Ghaemi, 2006). The newer atypical neuroleptics lack extrapyramidal effects as rigidity, tremor, bradykinesia, and akathesia which commonly occur with the first generation (Dubois, 2005).

Diagnose of antipsychotic overdoses can be through clinical manifestations symptoms and signs which include anticholinergic effects, extrapyramidal symptoms, neuroleptic malignant syndrome, seizures, and cardiac effects (Trenton et al., 2003). 
Antipsychotic overdose toxidromes should be differentiated from other toxic and non toxic causes as rhabdomyolysis, status epilepticus, toxicity of anticholinergic, antidepressant, and antihistamine (Pacher and Kecskemeti, 2004).

Antipsychotics rank in the top five substance classes involved in human exposures (Correll et al., 2006). Overdose of antipsychotic medication is more common among psychiatric patients than other individuals, although unintentional ingestion by children is not uncommon (Gussow, 2012).

\begin{abstract}
Aim of the Work
During the last few years, a large number of antipsychotic drug overdose cases were admitted to Menoufia Poison Control Center (MPCC) and the number of cases increases yearly so this work aims to study antipsychotic drug poisoning cases that were admitted to MPCC as regarding: socio demographic pattern, their clinical manifestation, investigation and outcome according to poisoning severity score.
\end{abstract}

\section{Patients, Material and Methods}

In this study, patients complaining of antipsychotic drug overdose admitted to MPCC from $1^{\text {st }}$ October 2012 to $30^{\text {th }}$ September 2013 were included. The study was carried out in an ethical manner following guidelines set by the Ethical Committee of Faculty of Medicine, Menoufia University. Informed valid consent was taken from the patients or from the patient's guardian and approval of head of MPCC before the study. Patients suffering from any systemic diseases and who didn't give informed consent were excluded from the study. The socio-demographic and clinical data were collected from each patient in a designed clinical toxicological sheet. Serum electrolytes, arterial blood gas, liver and kidney function tests, creatine phosphokinase (CPK) level were assessed. ECG was performed for each patient and was thoroughly analyzed. The studied cases were classified according to poisoning severity score. The clinical severity of poisoning was graded according to the method described by Persson et al., (1998) and developed by the International Program on Chemical Safety, the European Community, and the European Association of Poisons Centers and Clinical Toxicologists (IPCS/EC/EAPCCT) (Fruchtengarten, 2006). The poisoning severity score (PSS) is a fourscale grading as (0) none, (1) minor, (2) moderate, (3) severe, and (4) fatal. It was determined at the time of initial inquiry and following recovery, using examination findings including the Glasgow coma score, pupil size, convulsion, respiratory rate, pulse rate, blood pressure, body temperature, ECG findings, pain assessment, and laboratory presentations (serum glucose, potassium $(\mathrm{K})$, sodium $(\mathrm{Na})$, calcium $(\mathrm{Ca})$, $\mathrm{pH}$, and bicarbonate in arterial blood gases). The data collected were tabulated and analyzed by SPSS (statistical package for social science) version 17.0 on IBM compatible computer. Chi-square test $\left(\chi^{2}\right)$ was used to study association between two qualitative variables. P-value of $<0.05$ was considered statistically significant (Elliott and Woodward, 2007).

\section{Results}

Sex differentiation in age groups demonstrated in table (1) where the relation between sex and different age groups was significant $(\mathrm{P}=0.002)$. Females and males comprised $(33.5 \%)$ and $(25 \%)$ respectively in the age group 20-<40 years.

Table (2): Shows that there was highly significant relation between age and mode of poisoning as $\mathrm{P}=0.000$ and significant relation between gender and residence as regard to mode of poisoning where $\mathrm{P}$ $=0.03$ and 0.04 respectively. All cases under 10 years had taken the drug accidentally $(84.1 \%)$. The highest percent of cases committed suicide was in age group $20-<40(42.5 \%)$. Only one male case exposed to homicidal intake in age group $20-<40$ from urban area. Females and males constituted (77.5\%) and (73.7\%) for suicidal and accidental exposure respectively. The accidental mode was higher in rural side $(89.5 \%)$ of cases. On the contrary, suicide was $(75 \%)$ of cases from urban areas.

Table (3): shows that there was significant relation between PSS and co-ingestion, patients who were on psychic therapy and seasonal variation where $\mathrm{P}=0.048,0.004$ and 0.003 respectively. Regarding coingestion, $(60 \%)$ of cases were of moderate severity grade while one case $(100 \%)$ of fatal grade. Patients who were on psychic therapy and summer season predominance were $(100 \%)$ of cases of severe and fatal grades for each one. Also there was no significant relation between PSS and drug type (typical and atypical), delay time of presentation and mode of exposure where $\mathrm{P}=0.333,0.082$ and 0.786 respectively.

Table (4): Reveals significant relation between poisoning severity grades of the studied cases according to PSS and vomiting $(\mathrm{P}<0.01)$, where vomiting was presented in $(100 \%)$ of cases of fatal grade followed by $(73 \%)$ and $(60 \%)$ of cases were of minor and moderate grades respectively. Similar significant result was found with loss of consciousness, which it was of severe and fatal grades $(100 \%)$ for each followed by $(28 \%)$ of moderate severity grade. Regarding dizziness, it was represented minor and moderate grades $(53.3 \%)$ and $(8 \%)$ respectively. This relation was significant $(\mathrm{P}<0.01)$. Similar significant result $(\mathrm{P}<0.01)$ was found as regard convulsions where, severe and fatal severity grades were in $(100 \%)$ of cases for each, while $(48 \%)$ of cases were of moderate severity grade. Cases complained from palpitation $(60 \%)$ were of moderate grade. Abnormal movements represented moderate severity grade in $(60 \%)$ of cases. All cases $(100 \%)$ of the" none" grade of severity score had no complain. The relation between PSS and palpitation, abnormal movements and no complain was highly significant as $\mathrm{P}=0.00$.

Table (5): Shows a highly significant relation between PSS and ICU admission $(\mathrm{P}=0.000)$, where $(100 \%)$ of cases were of both severe and fatal grades while $(32 \%)$ of cases were of moderate grade. On the other hand, all of severe and fatal grades cases $(100 \%)$ and $(40 \%)$, and $(6.6 \%)$ of cases of moderate and minor grades respectively needed emergency stabilization in 
the form of supplemental oxygen, Coma Cocktail (Naloxon, Dextros, fluids and Thiamine) and intubation. The relation between PSS and emergency stabilization was significant as $\mathrm{P}=0.019$.

Table (6): Shows there was significant relation between serum potassium level and poisoning severity grades of the patients $(\mathrm{P}=0.002)$. Hypokalemia was in $(100 \%)$ of cases of both severe and fatal grades, meanwhile it was $(32 \%)$ and $(6.7 \%)$ of moderate and minor grades respectively. There was no significant relation between PSS and serum $\mathrm{Na}$, and $\mathrm{Ca}$ levels where $(\mathrm{P}=0.449$ and 0.575 respectively). As regards liver function tests, (100\%) of severe grades cases, presented with elevated liver function tests followed by (38.9\%), (26.7\%), and (20\%) in "none", minor and moderate grades respectively. On the other hand elevated renal function occurred in all severe and fatal severity grades cases. The relation was highly significant between PSS and liver and kidney function tests $(\mathrm{P}=0.001)$. Increased CPK level was in all cases of severe and fatal grades followed by $(12 \%)$ of cases in moderate severity grade. The relation between PSS and CPK level was significant as $(\mathrm{P}=0.05)$.

Frequency distribution of ECG changes among antipsychotic poisoned cases, ECG was normal in $(66.6 \%)$ of cases, on the other hand sinus tachycardia, wide QRS and depressed ST segment were $(20 \%),(6.6 \%)$ and $(6.6 \%)$ respectively as shown in table (7) and graphs $(1,2)$.
Table (8): Shows there was a highly significant relation between PSS and ECG changes $(\mathrm{P}=$ $0.000)$, where $(100 \%)$ of cases of both severe and fatal grades had sinus tachycardia and wide QRS respectively. Cases of moderate poisoning severity score had sinus tachycardia, prolonged QT and wide QRS (40\%, 16\% and 4\%) respectively.

Table (9): Shows highly significant relation between poisoning severity grades and mode of treatment as regards activated charcoal and sedatives where $\mathrm{P}=0.000$, all the studied cases of different poisoning grades received activated charcoal while $100 \%$ and $56 \%$ of severe and moderate poisoning grades respectively received sedatives. $\mathrm{NaHCO}_{3}$ given to cases of severe, moderate and minor poisoning severity grades $100 \%, 48 \%$ and $26.7 \%$, respectively. $100 \%$ of cases of severe and fatal PSS treated with vasopressor drugs. Cases of moderate poisoning severity grade $48 \%$ given Diphenhydramine. The relation between PSS and mode of treatment as regards to $\mathrm{NaHCO}_{3}$, Vasopressor and Diphenhydramine was significant as $\mathrm{P}=0.005,0.008$ and 0.032 respectively.

Table (10): Shows that there was highly significant relation between poisoning severity grades and outcome of the patients $(\mathrm{P}=0.000)$. Improvement occurred in $(100 \%),(48 \%),(38.9 \%)$ and $(26.7 \%)$ of severe, moderate, "none" and minor grades respectively. Only one case $(100 \%)$ died who had fatal severity grade.

Table 1: Statistical Analysis Chi-square $\left(\chi^{2}\right)$ of gender of studied cases versus age (No=60).

\begin{tabular}{|c|c|c|c|c|c|c|}
\hline \multirow{3}{*}{ Age in years } & \multicolumn{4}{|c|}{$\begin{array}{c}\text { Gender of studied group } \\
\text { No }=60\end{array}$} & \multirow{3}{*}{$\begin{array}{l}\text { Test of significance } \\
\chi^{2}\end{array}$} & \multirow{3}{*}{$P$ value } \\
\hline & \multicolumn{2}{|c|}{$\begin{array}{c}\text { Male } \\
\text { No }=24\end{array}$} & \multicolumn{2}{|c|}{$\begin{array}{c}\text { Female } \\
\text { No }=36\end{array}$} & & \\
\hline & No & $\%$ & No & $\%$ & & \\
\hline$<5$ yrs & 5 & 20.8 & 6 & 16.7 & & \\
\hline $5-<10 \mathrm{yrs}$ & 3 & 12.5 & 2 & 5.4 & & \\
\hline $10-<20 \mathrm{yrs}$ & 5 & 20.8 & 10 & 27.7 & 19.11 & $0.002 *$ \\
\hline $20-<40 \mathrm{yrs}$ & 6 & 25.0 & 12 & 33.5 & & \\
\hline$\geq 40 \mathrm{yrs}$ & 5 & 20.8 & 6 & 16.7 & & \\
\hline
\end{tabular}

$* P$ value $<0.05=$ significant, No= number of cases

Table 2: Statistical Analysis Chi-square $\left(\chi^{2}\right)$ and Fisher's Exact Test of the mode of exposure in correlation to the age, gender and residence of the studied cases $(\mathrm{No}=60)$.

\begin{tabular}{|c|c|c|c|c|c|c|c|c|}
\hline & \multicolumn{6}{|c|}{ Mode of exposure $\mathrm{No}=60$} & \multirow[t]{3}{*}{ Test of significance } & \multirow[t]{3}{*}{ P-value } \\
\hline & \multicolumn{2}{|c|}{$\begin{array}{c}\text { Accidental } \\
\text { No }=19\end{array}$} & \multicolumn{2}{|c|}{$\begin{array}{c}\text { Suicidal } \\
\text { No=40 }\end{array}$} & \multicolumn{2}{|c|}{$\begin{array}{c}\text { Homicidal } \\
\text { No }=1\end{array}$} & & \\
\hline & No & $\%$ & No. & $\%$ & No. & $\%$ & & \\
\hline $\begin{array}{r}\text { Age } \\
<5 \text { vears }\end{array}$ & 11 & 57.8 & 0 & 0 & 0 & 0 & \multirow{5}{*}{$\begin{array}{c}\chi^{2} \\
25.283\end{array}$} & \multirow{5}{*}{$0.000 * *$} \\
\hline $5-<10$ years & 5 & 26.3 & 0 & 0 & 0 & 0 & & \\
\hline $10-<20$ years & 2 & 10.6 & 13 & 32.5 & 0 & 0 & & \\
\hline $20-<40$ years & 0 & 0 & 17 & 42.5 & 1 & 100 & & \\
\hline$\geq 40$ years & 1 & 5.3 & 10 & 25.0 & 0 & 0 & & \\
\hline Gender & & & & & & & \multirow{3}{*}{$\begin{array}{c}\text { Fisher's Exact test } \\
2.297\end{array}$} & \multirow{3}{*}{$0.03 *$} \\
\hline Male & 14 & 73.7 & 9 & 22.5 & 1 & 100 & & \\
\hline Female & 5 & 26.3 & 31 & 77.5 & 0 & 0 & & \\
\hline Residence & & & & & & & \multirow{3}{*}{$\begin{array}{c}\chi^{2} \\
10.76\end{array}$} & \multirow{3}{*}{$0.04 *$} \\
\hline Rural & 17 & 89.5 & 10 & 25.0 & 0 & 0 & & \\
\hline Urban & 2 & 10.5 & 30 & 75.0 & 1 & 100 & & \\
\hline
\end{tabular}

$* P$ value $<0.05=$ significant,$* * P$ value $<0.001=$ highly significant, No $=$ number of cases 
Table 3: Statistical Analysis Chi-square $\left(\chi^{2}\right)$ of the relation between poison history and poisoning severity grades of the studied cases according to PSS (No=60).

\begin{tabular}{|c|c|c|c|c|c|c|c|c|c|c|c|c|}
\hline \multirow{3}{*}{ Poison history } & \multicolumn{10}{|c|}{$\begin{array}{c}\text { Poisoning Severity Score } \\
\text { No=60 }\end{array}$} & \multirow{3}{*}{$\begin{array}{c}\text { Test of } \\
\text { significance } \\
\chi^{2}\end{array}$} & \multirow{3}{*}{$\begin{array}{c}\mathbf{P} \\
\text { value }\end{array}$} \\
\hline & \multicolumn{2}{|c|}{$\begin{array}{c}\text { None } \\
\text { No }=18 \\
\end{array}$} & \multicolumn{2}{|c|}{$\begin{array}{c}\text { Minor } \\
\text { No }=15 \\
\end{array}$} & \multicolumn{2}{|c|}{$\begin{array}{c}\text { Moderate } \\
\text { No }=25 \\
\end{array}$} & \multicolumn{2}{|c|}{$\begin{array}{l}\text { Severe } \\
\text { No }=1 \\
\end{array}$} & \multicolumn{2}{|c|}{$\begin{array}{c}\text { Fatal } \\
\text { No }=1\end{array}$} & & \\
\hline & No & $\%$ & No & $\%$ & No & $\%$ & No & $\%$ & No & $\%$ & & \\
\hline $\begin{array}{l}\text { Drug type } \\
\text { Typical } \\
\text { Atypical } \\
\end{array}$ & $\begin{array}{c}4 \\
14 \\
\end{array}$ & $\begin{array}{l}22.2 \\
77.8 \\
\end{array}$ & $\begin{array}{c}3 \\
12 \\
\end{array}$ & $\begin{array}{l}20.0 \\
80.0 \\
\end{array}$ & $\begin{array}{c}9 \\
16 \\
\end{array}$ & $\begin{array}{l}36.0 \\
64.0 \\
\end{array}$ & $\begin{array}{l}1 \\
0 \\
\end{array}$ & $\begin{array}{c}100.0 \\
0 \\
\end{array}$ & $\begin{array}{l}1 \\
0\end{array}$ & $\begin{array}{c}100.0 \\
0 \\
\end{array}$ & 6.328 & 0.333 \\
\hline $\begin{array}{l}\text { Co- ingestion } \\
\text { Yes } \\
\text { No }\end{array}$ & $\begin{array}{c}8 \\
10 \\
\end{array}$ & $\begin{array}{l}44.4 \\
55.6 \\
\end{array}$ & $\begin{array}{l}8 \\
7\end{array}$ & $\begin{array}{l}53.3 \\
46.7 \\
\end{array}$ & $\begin{array}{l}15 \\
10\end{array}$ & $\begin{array}{l}60.0 \\
40.0\end{array}$ & $\begin{array}{l}0 \\
1\end{array}$ & $\begin{array}{c}0 \\
100.0\end{array}$ & $\begin{array}{l}1 \\
0\end{array}$ & $\begin{array}{c}100.0 \\
0\end{array}$ & 3.036 & $0.048 *$ \\
\hline $\begin{array}{l}\text { Delay of presentation } \\
<1 \mathrm{hr} \\
\geq 1 \mathrm{hr} \\
\end{array}$ & $\begin{array}{c}3 \\
15 \\
\end{array}$ & $\begin{array}{l}16.7 \\
83.3\end{array}$ & $\begin{array}{c}2 \\
13 \\
\end{array}$ & $\begin{array}{l}13.3 \\
86.7 \\
\end{array}$ & $\begin{array}{c}0 \\
25 \\
\end{array}$ & $\begin{array}{c}0 \\
100.0 \\
\end{array}$ & $\begin{array}{l}0 \\
1 \\
\end{array}$ & $\begin{array}{c}0 \\
100.0 \\
\end{array}$ & $\begin{array}{l}0 \\
1 \\
\end{array}$ & $\begin{array}{c}0 \\
100.0 \\
\end{array}$ & 4.582 & 0.082 \\
\hline $\begin{array}{l}\text { Mode of exposure } \\
\text { Accidental } \\
\text { Suicidal } \\
\text { Homicidal }\end{array}$ & $\begin{array}{c}5 \\
13 \\
0\end{array}$ & $\begin{array}{c}27.8 \\
72.2 \\
0\end{array}$ & $\begin{array}{c}2 \\
12 \\
1\end{array}$ & $\begin{array}{c}13.3 \\
80.0 \\
6.7\end{array}$ & $\begin{array}{c}5 \\
20 \\
0\end{array}$ & $\begin{array}{c}20.0 \\
80.0 \\
0\end{array}$ & $\begin{array}{l}0 \\
1 \\
0\end{array}$ & $\begin{array}{c}0 \\
100.0 \\
0\end{array}$ & $\begin{array}{l}0 \\
1 \\
0\end{array}$ & $\begin{array}{c}0 \\
100.0 \\
0\end{array}$ & 4.498 & 0.786 \\
\hline $\begin{array}{l}\begin{array}{c}\text { On antipsychotic } \\
\text { therapy }\end{array} \\
\text { Yes } \\
\text { No }\end{array}$ & $\begin{array}{c}6 \\
12 \\
\end{array}$ & $\begin{array}{l}33.3 \\
66.7 \\
\end{array}$ & $\begin{array}{c}3 \\
12 \\
\end{array}$ & $\begin{array}{l}20.0 \\
80.0 \\
\end{array}$ & $\begin{array}{c}4 \\
21 \\
\end{array}$ & $\begin{array}{l}16.0 \\
84.0 \\
\end{array}$ & $\begin{array}{l}1 \\
0 \\
\end{array}$ & $\begin{array}{c}100.0 \\
0 \\
\end{array}$ & $\begin{array}{l}1 \\
0 \\
\end{array}$ & $\begin{array}{c}100.0 \\
0 \\
\end{array}$ & 7.947 & $0.004 *$ \\
\hline $\begin{array}{l}\quad \text { Season } \\
\text { Summer } \\
\text { Spring } \\
\text { Autumn } \\
\text { Winter }\end{array}$ & $\begin{array}{l}10 \\
2 \\
2 \\
4\end{array}$ & $\begin{array}{l}55.6 \\
11.1 \\
11.1 \\
22.2\end{array}$ & $\begin{array}{l}6 \\
4 \\
3 \\
2\end{array}$ & $\begin{array}{l}40.0 \\
26.7 \\
20.0 \\
13.3\end{array}$ & $\begin{array}{c}11 \\
6 \\
6 \\
2\end{array}$ & $\begin{array}{c}44.0 \\
24.0 \\
24.0 \\
8.0\end{array}$ & $\begin{array}{l}1 \\
0 \\
0 \\
0\end{array}$ & $\begin{array}{c}100.0 \\
0 \\
0 \\
0\end{array}$ & $\begin{array}{l}1 \\
0 \\
0 \\
0\end{array}$ & $\begin{array}{c}100.0 \\
0 \\
0 \\
0\end{array}$ & 10.98 & $0.003 *$ \\
\hline
\end{tabular}

$* P$ value $<0.05=$ significant $P$ value $>0.05=$ non significant $N o=$ number of cases

Table 4: Statistical Analysis Chi-square $\left(\chi^{2}\right)$ of the relation between clinical manifestations and poisoning severity grades of the studied cases according to PSS (No=60).

\begin{tabular}{|c|c|c|c|c|c|c|c|c|c|c|c|c|}
\hline \multirow{3}{*}{$\begin{array}{c}\text { Clinical } \\
\text { manifestations }\end{array}$} & \multicolumn{10}{|c|}{ Poisoning Severity Score, $\mathrm{No}=60$} & \multirow{3}{*}{$\begin{array}{c}\text { Test of } \\
\text { significance } \\
\chi^{2}\end{array}$} & \multirow{3}{*}{$\begin{array}{c}\mathbf{P} \\
\text { value }\end{array}$} \\
\hline & \multicolumn{2}{|c|}{ None } & \multicolumn{2}{|c|}{ minor } & \multicolumn{2}{|c|}{ Moderate } & \multicolumn{2}{|c|}{ Severe } & \multicolumn{2}{|c|}{ Fatal } & & \\
\hline & No=18 & $\%$ & No=15 & $\%$ & No=25 & $\%$ & No $=1$ & $\%$ & No=1 & $\%$ & & \\
\hline $\begin{array}{l}\text { Vomiting } \\
\text { Yes } \\
\text { No } \\
\end{array}$ & $\begin{array}{c}1 \\
17 \\
\end{array}$ & $\begin{array}{c}5.6 \\
94.4 \\
\end{array}$ & $\begin{array}{c}11 \\
4 \\
\end{array}$ & $\begin{array}{l}73.3 \\
26.7 \\
\end{array}$ & $\begin{array}{l}15 \\
10 \\
\end{array}$ & $\begin{array}{l}60.0 \\
40.0 \\
\end{array}$ & $\begin{array}{l}0 \\
1\end{array}$ & $\begin{array}{c}0 \\
100 \\
\end{array}$ & $\begin{array}{l}1 \\
0\end{array}$ & $\begin{array}{c}100 \\
0\end{array}$ & 20.3 & $<0.01 *$ \\
\hline $\begin{array}{l}\text { Loss of } \\
\text { consciousness } \\
\text { Yes } \\
\text { No }\end{array}$ & $\begin{array}{c}0 \\
18\end{array}$ & $\begin{array}{c}0 \\
100\end{array}$ & $\begin{array}{c}1 \\
14\end{array}$ & $\begin{array}{c}6.7 \\
93.3\end{array}$ & $\begin{array}{c}7 \\
18\end{array}$ & $\begin{array}{l}28.0 \\
72.0\end{array}$ & $\begin{array}{l}1 \\
0\end{array}$ & $\begin{array}{c}100 \\
0\end{array}$ & $\begin{array}{l}1 \\
0\end{array}$ & $\begin{array}{c}100 \\
0\end{array}$ & 16.992 & $<0.01 *$ \\
\hline $\begin{array}{l}\text { Dizziness } \\
\text { Yes } \\
\text { No } \\
\end{array}$ & $\begin{array}{c}0 \\
18 \\
\end{array}$ & $\begin{array}{c}0 \\
100 \\
\end{array}$ & $\begin{array}{l}8 \\
7 \\
\end{array}$ & $\begin{array}{l}53.3 \\
46.7 \\
\end{array}$ & $\begin{array}{c}2 \\
23 \\
\end{array}$ & $\begin{array}{c}8.0 \\
92.0 \\
\end{array}$ & $\begin{array}{l}0 \\
1 \\
\end{array}$ & $\begin{array}{c}0 \\
100 \\
\end{array}$ & $\begin{array}{l}0 \\
1 \\
\end{array}$ & $\begin{array}{c}0 \\
100 \\
\end{array}$ & 19.872 & $<0.01 *$ \\
\hline $\begin{array}{l}\text { Convulsions } \\
\text { Yes } \\
\text { No }\end{array}$ & $\begin{array}{c}0 \\
18\end{array}$ & $\begin{array}{c}0 \\
100\end{array}$ & $\begin{array}{c}0 \\
15\end{array}$ & $\begin{array}{c}0 \\
100\end{array}$ & $\begin{array}{l}12 \\
13\end{array}$ & $\begin{array}{l}48.0 \\
52.0\end{array}$ & $\begin{array}{l}1 \\
0\end{array}$ & $\begin{array}{c}100 \\
0\end{array}$ & $\begin{array}{l}1 \\
0\end{array}$ & $\begin{array}{c}100 \\
0\end{array}$ & 25.118 & $<0.01 *$ \\
\hline $\begin{array}{l}\text { Palpitations } \\
\text { Yes } \\
\text { No }\end{array}$ & $\begin{array}{c}0 \\
18\end{array}$ & $\begin{array}{c}0 \\
100\end{array}$ & $\begin{array}{c}0 \\
15\end{array}$ & $\begin{array}{c}0 \\
100\end{array}$ & $\begin{array}{l}15 \\
10\end{array}$ & $\begin{array}{l}60.0 \\
40.0\end{array}$ & $\begin{array}{l}0 \\
1\end{array}$ & $\begin{array}{c}0 \\
100\end{array}$ & $\begin{array}{l}0 \\
1\end{array}$ & $\begin{array}{c}0 \\
100\end{array}$ & 28.0 & $0.00 * *$ \\
\hline $\begin{array}{l}\text { Abnormal } \\
\text { movements } \\
\text { Yes } \\
\text { No }\end{array}$ & $\begin{array}{c}0 \\
18\end{array}$ & $\begin{array}{c}0 \\
100\end{array}$ & $\begin{array}{c}0 \\
15\end{array}$ & $\begin{array}{c}0 \\
100\end{array}$ & $\begin{array}{l}15 \\
10\end{array}$ & $\begin{array}{l}60.0 \\
40.0\end{array}$ & $\begin{array}{l}0 \\
1\end{array}$ & $\begin{array}{c}0 \\
100\end{array}$ & $\begin{array}{l}0 \\
1\end{array}$ & $\begin{array}{c}0 \\
100\end{array}$ & 28.0 & $0.00 * *$ \\
\hline $\begin{array}{l}\text { No complaint } \\
\text { Yes } \\
\text { No }\end{array}$ & $\begin{array}{c}18 \\
0\end{array}$ & $\begin{array}{c}100 \\
0\end{array}$ & $\begin{array}{c}1 \\
14\end{array}$ & $\begin{array}{c}6.7 \\
93.3\end{array}$ & $\begin{array}{c}0 \\
25\end{array}$ & $\begin{array}{c}0 \\
100\end{array}$ & $\begin{array}{l}0 \\
1\end{array}$ & $\begin{array}{c}0 \\
100\end{array}$ & $\begin{array}{l}0 \\
1\end{array}$ & $\begin{array}{c}0 \\
100\end{array}$ & 55.68 & $0.00 * *$ \\
\hline
\end{tabular}

$* P$ value $<0.05=$ significant,$* * P$ value $<0.001=$ highly significant $P$ value $>0.05=$ non significant, No= cases number 
Table 5: Statistical Analysis Chi-square $\left(\chi^{2}\right)$ of the relation between poisoning severity grades in the studied cases according to PSS and their need for ICU admission and emergency stabilization.

\begin{tabular}{|c|c|c|c|c|c|c|c|c|c|c|c|c|}
\hline & \multicolumn{10}{|c|}{$\begin{array}{c}\text { Poisoning severity score } \\
\text { No }=60\end{array}$} & \multirow{3}{*}{$\begin{array}{c}\text { Test of } \\
\text { significance } \\
\chi^{2}\end{array}$} & \multirow{3}{*}{$P$ value } \\
\hline & \multicolumn{2}{|c|}{$\begin{array}{c}\text { None } \\
\text { No }=18 \\
\end{array}$} & \multicolumn{2}{|c|}{$\begin{array}{c}\text { Minor } \\
\text { No }=15\end{array}$} & \multicolumn{2}{|c|}{$\begin{array}{c}\text { Moderate } \\
\text { No = 25 }\end{array}$} & \multicolumn{2}{|c|}{$\begin{array}{c}\text { Severe } \\
\text { No=1 }\end{array}$} & \multicolumn{2}{|c|}{$\begin{array}{l}\text { Fatal } \\
\text { No=1 }\end{array}$} & & \\
\hline & No & $\%$ & No & $\%$ & No & $\%$ & No & $\%$ & No & $\%$ & & \\
\hline $\begin{array}{l}\text { ICU admission } \\
\text { Yes } \\
\text { No }\end{array}$ & $\begin{array}{c}0 \\
18 \\
\end{array}$ & $\begin{array}{c}0 \\
100.0 \\
\end{array}$ & $\begin{array}{c}0 \\
15 \\
\end{array}$ & $\begin{array}{c}0 \\
100.0 \\
\end{array}$ & $\begin{array}{c}8 \\
17 \\
\end{array}$ & $\begin{array}{l}32.0 \\
68.0 \\
\end{array}$ & $\begin{array}{l}1 \\
0 \\
\end{array}$ & $\begin{array}{c}100.0 \\
0 \\
\end{array}$ & $\begin{array}{l}1 \\
0 \\
\end{array}$ & $\begin{array}{c}100.0 \\
0 \\
\end{array}$ & 20.832 & $0.000 * *$ \\
\hline $\begin{array}{l}\text { Emergency } \\
\text { stabilization } \\
\text { Yes } \\
\text { No }\end{array}$ & $\begin{array}{c}0 \\
18\end{array}$ & $\begin{array}{c}0 \\
100.0\end{array}$ & $\begin{array}{c}1 \\
14\end{array}$ & $\begin{array}{c}6.6 \\
94.4\end{array}$ & $\begin{array}{l}10 \\
15\end{array}$ & $\begin{array}{l}40.0 \\
60.0 \\
\end{array}$ & $\begin{array}{l}1 \\
0\end{array}$ & $\begin{array}{c}100.0 \\
0\end{array}$ & $\begin{array}{l}1 \\
0\end{array}$ & $\begin{array}{c}100.0 \\
0\end{array}$ & 11.761 & $0.019 *$ \\
\hline
\end{tabular}

$* P$ value $<0.05=$ significant $* * P$ value $<0.001=$ highly significant No $=$ number of cases

Table 6: Statistical Analysis Chi-square $\left(\chi^{2}\right)$ of the relation between poisoning severity grades in the studied cases according to PSS and laboratory investigations (No=60).

\begin{tabular}{|c|c|c|c|c|c|c|c|c|c|c|c|c|}
\hline \multirow{3}{*}{$\begin{array}{l}\text { Laboratory } \\
\text { investigations }\end{array}$} & \multicolumn{10}{|c|}{$\begin{array}{c}\text { Poisoning severity score } \\
\text { No=60 }\end{array}$} & \multirow{3}{*}{$\begin{array}{c}\text { Test of } \\
\text { significance } \\
\chi^{2}\end{array}$} & \multirow{3}{*}{$P$ value } \\
\hline & \multicolumn{2}{|c|}{$\begin{array}{c}\text { None } \\
\text { No }=18\end{array}$} & \multicolumn{2}{|c|}{$\begin{array}{c}\text { Minor } \\
\text { No }=15\end{array}$} & \multicolumn{2}{|c|}{$\begin{array}{c}\text { Moderate } \\
\text { No }=\mathbf{2 5}\end{array}$} & \multicolumn{2}{|c|}{$\begin{array}{l}\text { Severe } \\
\text { No }=1\end{array}$} & \multicolumn{2}{|c|}{$\begin{array}{c}\text { Fatal } \\
\text { No }=1\end{array}$} & & \\
\hline & No & $\%$ & No & $\%$ & No & $\%$ & No & $\%$ & No & $\%$ & & \\
\hline \begin{tabular}{l}
\multicolumn{1}{c}{$\mathbf{N a}$} \\
Hyponatremia \\
Hypernatremia \\
Normal
\end{tabular} & $\begin{array}{c}0 \\
0 \\
18\end{array}$ & $\begin{array}{c}0 \\
0 \\
100.0\end{array}$ & $\begin{array}{c}0 \\
0 \\
15\end{array}$ & $\begin{array}{c}0 \\
0 \\
100.0\end{array}$ & $\begin{array}{c}3 \\
1 \\
21\end{array}$ & $\begin{array}{c}12.0 \\
4.0 \\
84.0\end{array}$ & $\begin{array}{l}1 \\
0 \\
0\end{array}$ & $\begin{array}{c}100.0 \\
0 \\
0\end{array}$ & $\begin{array}{l}1 \\
0 \\
0\end{array}$ & $\begin{array}{c}100.0 \\
0 \\
0\end{array}$ & 26.987 & 0.449 \\
\hline \begin{tabular}{l}
\multicolumn{1}{c}{$\mathbf{K}$} \\
Hypokalemia \\
Normal \\
\end{tabular} & $\begin{array}{c}0 \\
18 \\
\end{array}$ & $\begin{array}{c}0 \\
100.0 \\
\end{array}$ & $\begin{array}{c}1 \\
14 \\
\end{array}$ & $\begin{array}{c}6.7 \\
93.3 \\
\end{array}$ & $\begin{array}{c}8 \\
17\end{array}$ & $\begin{array}{l}32.0 \\
68.0 \\
\end{array}$ & $\begin{array}{l}1 \\
0 \\
\end{array}$ & $\begin{array}{c}100.0 \\
0\end{array}$ & $\begin{array}{l}1 \\
0\end{array}$ & $\begin{array}{c}100.0 \\
0 \\
\end{array}$ & 17.432 & $0.002 *$ \\
\hline \begin{tabular}{l}
\multicolumn{1}{c}{ Ca } \\
Hypocalcemia \\
Normal
\end{tabular} & $\begin{array}{c}0 \\
18\end{array}$ & $\begin{array}{c}0 \\
100.0\end{array}$ & $\begin{array}{c}0 \\
15\end{array}$ & $\begin{array}{c}0 \\
100.0\end{array}$ & $\begin{array}{c}2 \\
23\end{array}$ & $\begin{array}{c}8.0 \\
92.0\end{array}$ & $\begin{array}{l}0 \\
1\end{array}$ & $\begin{array}{c}0 \\
100.0\end{array}$ & $\begin{array}{l}0 \\
1\end{array}$ & $\begin{array}{c}0 \\
100.0\end{array}$ & 2.897 & 0.575 \\
\hline $\begin{array}{l}\text { Liver function tests } \\
\text { Increased } \\
\text { Decreased } \\
\text { Normal }\end{array}$ & $\begin{array}{c}7 \\
0 \\
11 \\
\end{array}$ & $\begin{array}{c}38.9 \\
0 \\
61.1 \\
\end{array}$ & $\begin{array}{c}4 \\
0 \\
10 \\
\end{array}$ & $\begin{array}{c}26.7 \\
0 \\
73.3 \\
\end{array}$ & $\begin{array}{c}5 \\
0 \\
20 \\
\end{array}$ & $\begin{array}{c}20.0 \\
0 \\
80.0 \\
\end{array}$ & $\begin{array}{l}1 \\
0 \\
0\end{array}$ & $\begin{array}{c}100.0 \\
0 \\
0 \\
\end{array}$ & $\begin{array}{l}0 \\
0 \\
1 \\
\end{array}$ & $\begin{array}{c}0 \\
0 \\
100.0 \\
\end{array}$ & 7.844 & $0.001 * *$ \\
\hline $\begin{array}{l}\text { Kidney function test } \\
\text { Elevated } \\
\text { Normal }\end{array}$ & $\begin{array}{c}2 \\
16 \\
\end{array}$ & $\begin{array}{l}11.1 \\
88.9\end{array}$ & $\begin{array}{c}0 \\
15 \\
\end{array}$ & $\begin{array}{c}0 \\
100.0\end{array}$ & $\begin{array}{c}2 \\
23\end{array}$ & $\begin{array}{c}8.0 \\
92.0\end{array}$ & $\begin{array}{l}1 \\
0\end{array}$ & $\begin{array}{c}100.0 \\
0\end{array}$ & $\begin{array}{l}1 \\
0\end{array}$ & $\begin{array}{c}100.0 \\
0\end{array}$ & 19.802 & $0.001 * *$ \\
\hline $\begin{array}{l}\text { CPK } \\
\text { Normal } \\
\text { Increased } \\
\end{array}$ & $\begin{array}{c}18 \\
0\end{array}$ & $\begin{array}{c}100.0 \\
0\end{array}$ & $\begin{array}{c}15 \\
0\end{array}$ & $\begin{array}{c}100.0 \\
0\end{array}$ & $\begin{array}{c}22 \\
3\end{array}$ & $\begin{array}{l}88.0 \\
12.0\end{array}$ & $\begin{array}{l}0 \\
1\end{array}$ & $\begin{array}{c}0 \\
100.0\end{array}$ & $\begin{array}{l}0 \\
1\end{array}$ & $\begin{array}{c}0 \\
100.0\end{array}$ & 30.207 & $0.05 *$ \\
\hline
\end{tabular}

$* P$ value $<0.05=$ significant, $* * P$ value $<0.001=$ highly significant $P$ value $>0.05=$ non-significant No= cases number

Table 7: Frequency distribution of ECG changes of the studied cases $(\mathrm{No}=60)$.

\begin{tabular}{|l|c|c|}
\hline \multicolumn{1}{|c|}{ ECG changes } & No. & \% \\
\hline Normal & 40 & 66.6 \\
\hline Wide QRS & 4 & 6.7 \\
\hline Depressed ST segment & 4 & 6.7 \\
\hline Sinus tachycardia & 12 & 20.0 \\
\hline Total & 60 & 100.0 \\
\hline
\end{tabular}


Table 8: Statistical Analysis Chi-square $\left(\chi^{2}\right)$ of the relation between poisoning severity grades in the studied cases according to PSS and ECG changes $(\mathrm{No}=60)$.

\begin{tabular}{|c|c|c|c|c|c|c|c|c|c|c|c|c|}
\hline \multirow{3}{*}{ ECG changes } & \multicolumn{10}{|c|}{$\begin{array}{c}\text { Poisoning Severity Score } \\
\text { No=60 }\end{array}$} & \multirow{3}{*}{$\begin{array}{l}\text { Test of significance } \\
\qquad \chi^{2}\end{array}$} & \multirow{3}{*}{$P$ value } \\
\hline & \multicolumn{2}{|c|}{$\begin{array}{c}\text { None } \\
\text { No }=18\end{array}$} & \multicolumn{2}{|c|}{$\begin{array}{l}\text { Minor } \\
\text { No }=15\end{array}$} & \multicolumn{2}{|c|}{$\begin{array}{l}\text { Moderate } \\
\text { No }=25\end{array}$} & \multicolumn{2}{|c|}{$\begin{array}{l}\text { Severe } \\
\text { No }=1\end{array}$} & \multicolumn{2}{|c|}{$\begin{array}{c}\text { Fatal } \\
\text { No }=1\end{array}$} & & \\
\hline & No & $\%$ & No & $\%$ & No & $\%$ & No & $\%$ & No & $\%$ & & \\
\hline Normal & 18 & 100.0 & 12 & 80.0 & 10 & 40.0 & 0 & 0 & 0 & 0 & & \\
\hline Wide QRS & 0 & 0 & 2 & 13.3 & 1 & 4.0 & 0 & 0 & 1 & 100.0 & 51421 & 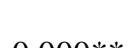 \\
\hline Prolonged QT & 0 & 0 & 0 & 0 & 4 & 16.0 & 0 & 0 & 0 & 0 & 51.431 & $0.000^{* * *}$ \\
\hline Sinus tachycardia & 0 & 0 & 1 & 6.7 & 10 & 40.0 & 1 & 100.0 & 0 & 0 & & \\
\hline
\end{tabular}

** $P$ value $<0.001=$ highly significant $N o=$ number of cases

Table 9: Statistical Analysis Chi-square $\left(\chi^{2}\right)$ of the relation between poisoning severity grades in the studied cases according to PSS and mode of treatment (No=60).

\begin{tabular}{|c|c|c|c|c|c|c|c|c|c|c|c|c|}
\hline \multirow{3}{*}{ Mode of treatment } & \multicolumn{10}{|c|}{$\begin{array}{c}\text { Poisoning severity score } \\
\text { No=60 } \\
\end{array}$} & \multirow{3}{*}{$\begin{array}{c}\text { Test of } \\
\text { significance } \\
\chi^{2}\end{array}$} & \multirow{3}{*}{$P$ value } \\
\hline & \multicolumn{2}{|c|}{$\begin{array}{c}\text { None } \\
\text { No }=18\end{array}$} & \multicolumn{2}{|c|}{$\begin{array}{c}\text { Minor } \\
\text { No }=15\end{array}$} & \multicolumn{2}{|c|}{$\begin{array}{c}\text { Moderate } \\
\text { No }=25\end{array}$} & \multicolumn{2}{|c|}{$\begin{array}{l}\text { Severe } \\
\text { No }=1\end{array}$} & \multicolumn{2}{|c|}{$\begin{array}{c}\text { Fatal } \\
\text { No }=1\end{array}$} & & \\
\hline & No & $\%$ & No & $\%$ & No & $\%$ & No & $\%$ & No & $\%$ & & \\
\hline $\begin{array}{l}\text { Activated charcoal } \\
\text { Yes } \\
\text { No }\end{array}$ & $\begin{array}{c}18 \\
0\end{array}$ & $\begin{array}{c}100.0 \\
0\end{array}$ & $\begin{array}{c}15 \\
0\end{array}$ & $\begin{array}{c}100.0 \\
0\end{array}$ & $\begin{array}{c}25 \\
0\end{array}$ & $\begin{array}{c}100.0 \\
0\end{array}$ & $\begin{array}{l}1 \\
0\end{array}$ & $\begin{array}{c}100.0 \\
0\end{array}$ & $\begin{array}{l}0 \\
1\end{array}$ & $\begin{array}{c}0 \\
100.0\end{array}$ & 60.000 & $0.000 * *$ \\
\hline $\begin{array}{l}\text { Sodium bicarbonate } \\
\quad\left(\mathrm{NaHCO}_{3}\right) \\
\text { Yes } \\
\text { No }\end{array}$ & $\begin{array}{c}0 \\
18\end{array}$ & $\begin{array}{c}0 \\
100.0\end{array}$ & $\begin{array}{l}4 \\
11\end{array}$ & $\begin{array}{l}26.7 \\
73.3\end{array}$ & $\begin{array}{l}12 \\
13\end{array}$ & $\begin{array}{l}48.0 \\
52.0\end{array}$ & $\begin{array}{l}1 \\
0\end{array}$ & $\begin{array}{c}100.0 \\
0\end{array}$ & $\begin{array}{l}0 \\
1\end{array}$ & $\begin{array}{c}0 \\
100.0\end{array}$ & 14.824 & $0.005^{*}$ \\
\hline $\begin{array}{l}\text { Vasopressors } \\
\text { Yes } \\
\text { No }\end{array}$ & $\begin{array}{c}2 \\
16\end{array}$ & $\begin{array}{l}11.1 \\
88.9\end{array}$ & $\begin{array}{c}1 \\
14\end{array}$ & $\begin{array}{c}6.7 \\
93.3\end{array}$ & $\begin{array}{c}3 \\
22\end{array}$ & $\begin{array}{l}12.0 \\
88.0\end{array}$ & $\begin{array}{l}1 \\
0\end{array}$ & $\begin{array}{c}100.0 \\
0\end{array}$ & $\begin{array}{l}1 \\
0\end{array}$ & $\begin{array}{c}100.0 \\
0\end{array}$ & 13.692 & $0.008 *$ \\
\hline $\begin{array}{ll} & \text { Sedatives } \\
\text { Yes } & \\
\text { No } & \end{array}$ & $\begin{array}{c}1 \\
17\end{array}$ & $\begin{array}{c}5.6 \\
94.4\end{array}$ & $\begin{array}{c}1 \\
14\end{array}$ & $\begin{array}{c}6.7 \\
93.3\end{array}$ & $\begin{array}{l}14 \\
11\end{array}$ & $\begin{array}{l}56.0 \\
44.0\end{array}$ & $\begin{array}{l}1 \\
0\end{array}$ & $\begin{array}{c}100.0 \\
0\end{array}$ & $\begin{array}{l}0 \\
1\end{array}$ & $\begin{array}{c}0 \\
100.0\end{array}$ & 4.0 & $0.000 * *$ \\
\hline $\begin{array}{l}\text { Diphenhydramine } \\
\text { Yes } \\
\text { No }\end{array}$ & $\begin{array}{c}3 \\
15\end{array}$ & $\begin{array}{l}16.7 \\
83.3\end{array}$ & $\begin{array}{c}1 \\
14\end{array}$ & $\begin{array}{c}6.7 \\
93.3\end{array}$ & $\begin{array}{l}12 \\
13\end{array}$ & $\begin{array}{l}48.0 \\
52.0\end{array}$ & $\begin{array}{l}0 \\
1\end{array}$ & $\begin{array}{c}0 \\
100.0\end{array}$ & $\begin{array}{l}0 \\
1\end{array}$ & $\begin{array}{c}0 \\
100.0\end{array}$ & 10.534 & $0.032 *$ \\
\hline
\end{tabular}

$* P$ value $<0.05=$ significant,$* * P$ value $<0.001=$ highly significant, No $=$ number of cases

Table 10: Statistical Analysis Chi-square $\left(\chi^{2}\right)$ of the relation between poisoning severity grades in the studied cases according to PSS and outcome (No=60).

\begin{tabular}{|c|c|c|c|c|c|c|c|c|c|c|c|c|}
\hline \multirow{3}{*}{ Outcome } & \multicolumn{10}{|c|}{ Poisoning severity score, $\mathrm{No}=60$} & \multirow{3}{*}{$\begin{array}{c}\text { Test of } \\
\text { significance } \\
\chi^{2}\end{array}$} & \multirow{3}{*}{$P$ value } \\
\hline & \multicolumn{2}{|c|}{$\begin{array}{c}\text { None } \\
\text { No }=18\end{array}$} & \multicolumn{2}{|c|}{$\begin{array}{c}\text { Minor } \\
\text { No }=15\end{array}$} & \multicolumn{2}{|c|}{$\begin{array}{l}\text { Moderate } \\
\text { No }=25\end{array}$} & \multicolumn{2}{|c|}{$\begin{array}{c}\text { Severe } \\
\text { No=1 }\end{array}$} & \multicolumn{2}{|c|}{$\begin{array}{l}\text { Fatal } \\
\text { No }=1\end{array}$} & & \\
\hline & No & $\%$ & No & $\%$ & No & $\%$ & No & $\%$ & No & $\%$ & & \\
\hline Improved & 7 & 38.9 & 4 & 26.7 & 12 & 48.0 & 1 & 100.0 & 0 & 0 & \multirow{4}{*}{65.848} & \multirow{4}{*}{$0.000 * *$} \\
\hline $\begin{array}{l}\text { Discharged on } \\
\text { request }\end{array}$ & 7 & 38.9 & 5 & 33.3 & 4 & 16.0 & 0 & 0 & 0 & 0 & & \\
\hline escaped & 4 & 22.2 & 6 & 40.0 & 9 & 36.0 & 0 & 0 & 0 & 0 & & \\
\hline dead & 0 & 0 & 0 & 0 & 0 & 0 & 0 & 0 & 1 & 100.0 & & \\
\hline
\end{tabular}

$* * P$ value $<0.001=$ highly significant $N o=$ number of cases.

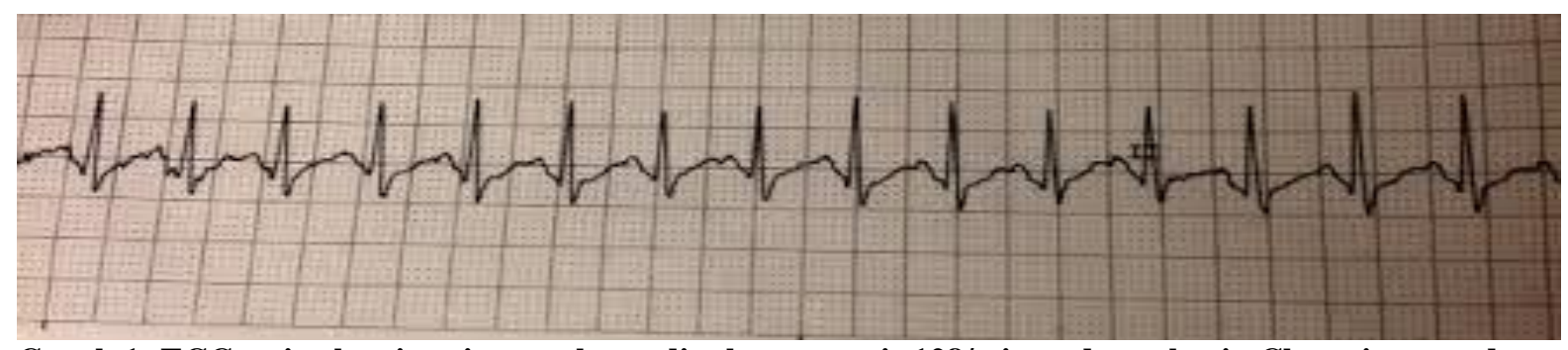

Graph 1: ECG strip showing sinus tachycardia: heart rate is 128/min and regular in Clozapine overdose. 


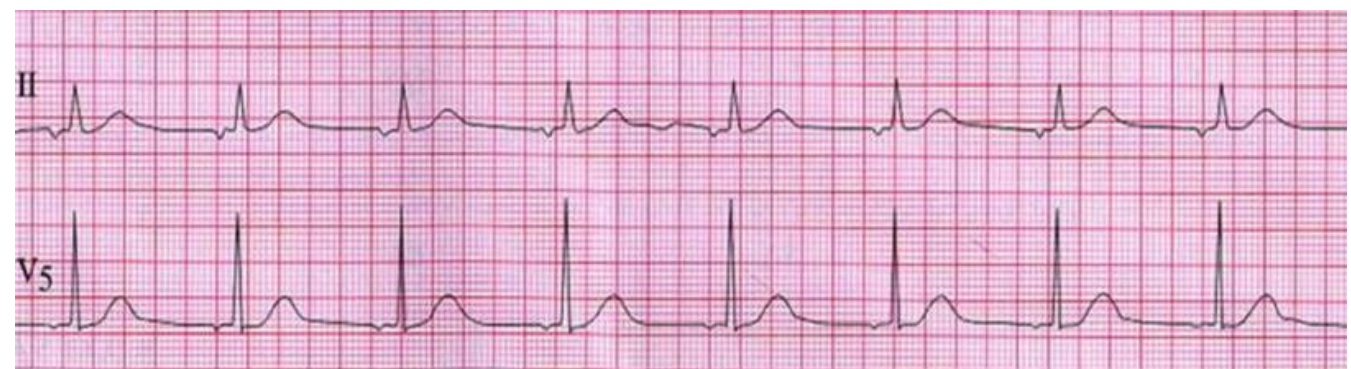

Graph 2: ECG strip showing wide QRS complex (0.14 s) in a case of Haloperidol overdose (normal QRS complex is $<3$ small squares i.e. $<0.12 \mathrm{~s}$ ).

\section{Discussion}

Poisoning occurs in all regions and countries and represents a leading cause of death in some countries (Bohnert et al., 2010).

Drug overdose is one of the easiest methods of attempting suicide, which is one of the major health problems world-wide (Aslan et al., 2011).

Poisoning due to medical drugs is a major problem in children as well as adults (Arokiasamy, 1994). The study showed that females outnumbered males. This result agreed with Doshi et al., (2005) who found that female to male ratio was $1.7: 1.3$. On the contrary to this study Soueif, (1994) found that poisoning was more common among males than females to cope with psychosocial problems or difficult working conditions, as well as at times of studying and examinations. Adejuyigbe et al., (2002) also found that poisoning was more common among males than females.

Males and females comprised the predominance of age group $20-<40$ years. This coincides with Batra et al., (2003) who stated that the most affected age group in their study in rural India was 21-30 years, followed by group 31-40 years. Gupta and Vaghela (2005) stated that maximum cases of poisoning in Jamnagar, India belonged to second and third decades of life.

The majority of cases under 10 years old had taken the overdose accidentally it could be due to that this age has increased activities and skills causing more exposure to their environment (Kliegman et al., 2008). Gupta et al., (2003) stated that children lack discrimination between harmful and non harmful things. Infants and small children are closer to the ground than older children; in addition, children have a natural exploratory curiosity and put everything in their mouth. Predominance of males in accidental poisoning due to that males are more active and tend to have more exploratory character than females in childhood (Malangu, 2008).

The results showed that the suicidal mode of poisoning was $(66.6 \%)$ of cases. The majority of cases were females $(75 \%)$. These results agreed with Garg and Verma, (2010) who stated that suicidal mode was the most common mode of acute intoxication. Yasan et al., (2008) found that higher suicide attempt rates were observed in females compared to the males. Suicidal attempts in antipsychotic overdose cases can be explained by the presence of depressive symptoms including depressed mood and hopelessness, stressful life events, divorce, separation and presence of delusions and/or auditory hallucinations (Bouhlel et al ., 2012). Other reported reasons of suicide attempts were impulsive suicides which are generally motivated by anger, the desire to get even, the wish to frighten or punish others, or the need to avoid intense shame subsequent to economic crisis, examination failure, love failure, quarrels, or unemployment, and chronic illness (Aslan et al., 2011). In the current study there was only one homicidal case it was for a father in the third decade from urban area was given (risperidal) in his tea by his daughter.

Accidental poisoning was higher in rural areas $(89.5 \%)$, while suicidal poisoning was found more in urban areas $(75 \%)$. This result was in accordance with the results reported by Cahfer and Ismail, (2004); Anthony and Kulkarni, (2012). Rural nature of Menoufia governorate and low socioeconomic standards associated with low levels of education and culture, easy availability, extensive use and low cost of the drugs, all make the population more vulnerable for accidental poisoning. The same result was reported by Garg and Verma, (2010). This was against Manzar et al., (2010) who reported that persons in urban areas are more exposed than those in rural areas due to mothers are workers leading to neglect of child.

The results revealed that there was significant relation between severity grades of the studied cases according to PSS and co-ingestion of drugs with antipsychotics. This coincided with Olson (2012) who stated that it is not uncommon for patients to take multiple psychiatric medications and toxicity of antipsychotic medications may be increased by coingestion of other agents, particularly drugs with similar metabolic pathways such as lithium, cyclic or other antidepressants and benzodiazepines.

Also there was significant relation between PSS and patients who were on psychic therapy. This result was in accordance with Aslan et al., (2011) who found that $(22.5 \%)$ of the patients had previous visits to the psychiatry outpatient clinics prior to the admission to the emergency department for intoxication.

Summer represented the commonest season for antipsychotic overdose cases. The rate of cases is higher during summer due to the results of end year examinations appear for secondary schools and universities and suicidal attempts increase due to failure of examinations for fearing of guilt or to escape punishment. Also children on summer holidays are 
more likely to be outdoors or to be left at home unattended or in the care of an older child or elderly relatives (Adejuyigbe et al., 2002). This result was in accordance with that published by Oguche et al., (2007) as they stated that highest frequency of admission was recorded during hot and dry months of March to June.

Concerning clinical manifestations, the results revealed vomiting was evident in $(100 \%)$ of fatal severity grade cases followed by $(73 \%)$ in minor grade cases. Vomiting can be explained by the anticholinergic effects of antipsychotics (Lackey, 2012). Cases of loss of consciousness were of severe and fatal grades followed by $(28 \%)$ of cases of moderate grade. This could be explained by the centrally mediated sedation and diminished cerebral perfusion secondary to systemic hypotension and anticholinergic effects contributed to CNS depression caused by antipsychotics (Lackey, 2012). Kwiatkowsk, (2008) stated that mental state changes were the most common manifestation in antipsychotic overdose. Impaired consciousness is a common and dose dependent feature of antipsychotic overdose, ranging from somnolence to frank coma. Concerning dizziness, it was evident in minor grade cases $(53.3 \%)$ followed by moderate grade cases (8\%). Hoffman et al., (2007) found that drowsiness and dizziness were the most common clinical features. Also blockage of peripheral $\alpha 1$ adrenergic leads to decreased vasomotor tone and accounts for it.

Convulsions were found in $(100 \%)$ of severe and fatal grades and $(48 \%)$ for moderate grade. Levine and Ruha, (2012) stated that the typical and atypical antipsychotics have the ability to lower seizure threshold. As regard palpitation, it was presented in $(60 \%)$ of moderate grade cases. Palpitation may reflect the anti cholinergic effect of the drug and reflex tachycardia can be due to hypotension, patient's pain and anxiety (Minns and Clark, 2012).

Abnormal movements were found in $(60 \%)$ of moderate grade cases. Levine and Ruha, (2012) reported that Extrapyramidal syndromes can occur with either typical or atypical antipsychotics. Patients who had no complaints were recorded in $(100 \%)$ of "none" grade cases. This was in accordance with Zakaria et al., (2012) who reported that although overdose and poisoning by antipsychotics are common, most cases with pure overdose manifest no symptoms or only mild toxicity. This finding can be explained by allegation of taking the drug or the patients come within one hour of ingestion or the parents may doubt their children taking the drug and they bring them for assurance or the patients may had induced vomiting by parents, friend or one of their relatives.

In the present study, cases admitted to ICU were of the severe and fatal grades $(100 \%)$ and $(32 \%)$ of moderate grade. These cases had one or more of the clinical criteria for ICU admission in poisoned patients; these criteria are: $\mathrm{PCO} 2>45 \mathrm{mmHg}$, need for emergency intubation, the presence of post ingestion seizures, unresponsiveness to verbal stimuli, a nonsinus cardiac rhythm, second- or third-degree AV block, systolic blood pressure less than $80 \mathrm{mmHg}$, or
QRS duration $\geq 0.12 \mathrm{~S}$ (Steven, 2006). Regarding the need for emergency stabilization, the study showed that all severe and fatal grades cases and (40\%) of moderate grade needed emergency stabilization. Hoffman et al., (2007) stated that indication of emergency stabilization in the form of supplemental oxygen should be administered if hypoxia is present, and patients with altered mental status should receive thiamine, naloxone, and parenteral dextrose as needed. Intubation and ventilation are rarely required, but may be necessary for patients with very large overdoses of antipsychotics or ingestion of other CNS depressants.

The results showed that $(100 \%)$ of cases of severe and fatal grades toxicity had hypokalemia. Alfonzo et al., (2006) stated that potassium disorders are common and may precipitate cardiac arrhythmias or cardiopulmonary arrest. Liver functions levels were elevated in $(100 \%),(26.7 \%)$, and $(20.0 \%)$ of severe, minor and moderate grades toxicity cases respectively. This was due to co-ingested drugs such as acetaminophen, benzodizapines and antidepressants. This is in accordance with Pratt and Kaplan, (2000). Elevation of renal functions levels in $(10 \%)$ from all studied cases in this study could be explained by the fact that elder population on antipsychotics was susceptible to diminished renal function; thus having high intoxication risk. The results showed that increased CPK level in all cases of severe and fatal toxicity grades and in (12\%) of cases of moderate grade. This is in agreement with Saltik and Basgul, (2012) who reported that numerous cases of Neuro Malignant Syndrome (NMS) have been reported with atypical antipsychotic medications including clozapine, olanzapine, quetiapine, and risperidone in recent years. On contrary to Strawn et al., (2007) ; Seitz and Gill, (2009) reported that NMS most often associated with typical high-potency neuroleptics e.g. haloperidol, fluphenazine.

The results showed that the frequency of ECG changes of studied cases was sinus tachycardia (20\%) followed by wide QRS and prolonged QT and depressed ST segment (6.7\%) for each. This result was in agreement with Minns and Clark, (2012) who reported that the most common cardiovascular effects that occur after atypical antipsychotic overdose are tachycardia, mild hypotension, and prolongation of the QT interval. There was a highly significant relation between PSS and ECG changes, Zareba and Lin, (2003) stated that the risk of QT prolongation and resultant torsades de pointes is dose dependent and slightly increased in overdose. The study showed that all the studied cases received activated charcoal, Vernon and Gleich, (1997) reported that activated charcoal remains the gastrointestinal decontamination method of choice. Repeated doses of activated charcoal every 6 hours without cathartics may increase clearance of some neuroleptics that undergo enterohepatic circulation (Bond, 2002). Sedatives were used in $28.3 \%$ of cases to control convulsions, this in accordance with Wills and Erickson, (2005) who stated that multiple or refractory seizures can be treated with benzodiazepines. The cardiotoxic effects of antipsychotic were treated with sodium biocarbonate in 
$28.3 \%$ of cases. Its efficacy has been attributed to counteracting the sodium channel blockade. Serum alkalization favors dissociation of the drug from myocardial $\mathrm{Na}+$ channels and the extra cellular $\mathrm{Na}+$ load improves $\mathrm{Na}+$ channel function (Alkatib et al., 2003). Vasopressors were used in $13.3 \%$ of cases who sustained hypotensive and manifested with signs of cardiogenic shock. Vasoconstrictor agents stimulate beta1- and alpha-adrenergic receptors, which, in turn increases cardiac muscle contractility, heart rate, and vasoconstriction. As a result, systemic blood pressure and coronary blood-flow increases (Shy and Rund 2011). Cases (26.7\%) were treated with Diphenhydramine, anticholinergic medications help restore balance between dopaminergic and cholinergic neurotransmission (Goldfrank et al., 2007).

The study showed that mortality is relatively rare with overdose of antipsychotic medication as it was $(1.6 \%)$ of total number of cases. This concurred with the result of Huybrechts et al., (2012) who reported that ingestions fatalities remain quite uncommon, with a case fatality rate of approximately $0.02 \%$ of all reported exposures because of the high toxic-therapeutic ratio.

\section{Conclusion}

Significant relation was found between PSS and poison history as regard co-ingestion and patients on antipsychotic therapy. Cases with moderate severity toxicity had palpation and abnormal movements. Acute antipsychotic overdose seldom results in death.

\section{Recommendation}

Increasing physician awareness regarding appropriate pharmacologic treatment of psychiatric problems and patient education about the benefits and possible side effects of antipsychotic medication.

\section{References}

Adejuyigbe E, Onayade A and Senbanjo I (2002): Childhood poisoning at the Obafemi Awolowo University Teaching Hospital, IleIfe, Nigeria, Niger J Med. 11(4):183-6.

Alfonzoa A, Isles C, Geddes C et al., (2006): Potassium disorders: Clinical spectrum and emergency management. Resuscitation. 70: 10-25.

AlKhatib S, Alle N, Kramer J et al., (2003): What clinicians should know about the QT interval. JAMA. 289: 2120-212.

Ananth J, Parameswaran S, Gunatilake S, et al., (2004): Neuroleptic malignant syndrome and atypical antipsychotic drugs. J Clin Psychiatry. Apr 65(4):464-470.

Anthony L and Kulkarni C (2012):Patterns of poisoning and drug overdose and their outcome among in-patients admitted to the emergency medicine department of a tertiary care hospital.Indian J Crit Care Med.16:130-5.

Arokiasamy J (1994): Accidental poisoning: selected aspects of its epidemiology and prevention. Med J Malaysia. 49 (2): 109-112.

Aslan S, Emet M, Cakir Z et al., (2011): Suicide attempts with amitriptyline in adults: A prospective, demographic, clinical study. Turk J Med Sci. 41 (2): 243-249.

Batra A, Keoliya A and Jadhav G (2003): Poisoning: An unnatural cause of morbidity and mortality in rural India. JAPI. 51: 955-959.

Bohnert A, Fudalej S, and Ilgen M (2010): Increasing poisoning mortality rates in the United States, 1999-2006 Public Health Rep. 125 (4): 542547.

Bond G (2002): The role of activated charcoal and gastric emptying in gastrointestinal decontamination. Ann Emerg Med. 39 (3): 273-286.

Bouhlel S, M'solly M, Benhawala S et al., (2012): Factors related to sucide attempts in a Tunisian sample of patients with schizophrenia- 39 (1): 6-12. In: www.ncbi.nlm.nih.gov/pubmed

Cahfer G and Ismail H (2004): Cases of acute poisoning in Southeasr Anatolia of Turkey. Cilt. 31 (2): 37-45.

Correll C, Penzner J, Parikh U et al., (2006): Recognizing and monitoring adverse events of second-generation antipsychotics in children and adolescents. Child Adolesc Psychiatr Clin N Am. 15 (1):177-206.

Doshi A, Boudreaux E, Wang N et al., (2005): National study of US emergency department visits for attempted suicide and self-inflicted injury, 1997-2001. Ann Emerg Med. 46: 369-75.

Dubois D (2005): Toxicology and overdose of atypical antipsychotic medications in children: Does newer necessarily mean safer? Curr Opin Pediatr. 17:227-233.

Elliott A and Woodward W (2007): SPSS (Statistical package of the social sciences, SAGE puplications. California, London and New Delhi, printed in USA.

Frunchtengarten L (2006): Handling poison information, poison prevention week May 28 to June 4, Kingston, Jamaica, data show.

Garg V and Verma S (2010): Original research paper trends of poisoning in rural area of south-west. Punjab,J Indian Acad Forensic Med. 32 (3): 189-193.

Goldfrank L, Hoffman R, Nelson L et al., (2007): in Goldfrank's manual of toxicologic emergencies. $8^{\text {th }}$ ed., McGraw-Hill. New York. Ch 67. pp. 583-590.

Gupta B and Vaghela P (2005): Profile of fatal poisoning in and around Jamnagar. Journal of Indian Academy of Forensic Medicine. 27(3): 145-148.

Gupta S, Peshin S, Srivastava A et al., (2003): A study of childhood poisoning at National Poisons Information Centre, All India Institute of Medical Sciences, New Delhi. Journal of Occupational Health. 45: 191-196.

Gussow L (2012): Atypical antipsychotic overdose: It is all about the receptors www.EM-News.com

Haddad P and Dursun S (2008): Neurological complications of psychiatric drugs: Clinical 
features and management. Hum Psychopharmacol. 23 (1):15-26.

Hoffman S, Nelson M, Howland A, et al., (2007): in Goldfrank's manual of toxicologic emergencies. $8^{\text {th }}$ ed., McGraw-Hill. New York.Ch 67. pp. 583-590.

Huybrechts K, Gerhard T, Crystal S et al., (2012): Differential risk of death in older residents in nursing homes prescribed specific antipsychotic drugs: Population based cohort study. BMJ. 344:e977.

Kliegman R, Behrman R, Jenson $\mathrm{H}$ et al., (2008): Nelson textbook of pediatrics, $18^{\text {th }}$ ed., Philadelphia USA.Ch 52,53,54,55 and 60. pp. 267-319.

Kwiatkowsk T (2008): Antipsychotic agents. In: Handbook of Medical Toxicology. Peter Vicceellio. Little and Brown Company. Boston/Toronto/London. Ch59. pp. 528-539.

Lackey D (2012): Antipsychotic drugs including phenothiazines. In: Olson's Poisoning and drug overdose by Olson K R et al., $6^{\text {th }}$ edition, Section II, pgs: 200-5.San Francisco, California.

Levine M and Ruha A (2012): Overdose of atypical antipsychotics: Clinical presentation, mechanisms of toxicity and management. CNS Drugs. 26 (7):601-11.

Malangu M (2008): Acute poisoning at two hospitals in Kampala-Uganda.J Forensic Leg Med. 15 (8): 489-92.

Manzar N, Saad S, Manzar B et al., (2010): The study of etiological and demographic characteristics of acute household accidental poisoning in children: A consecutive case series study from Pakistan. Research article Published: 3 May 2010.

Minns A and Clark R (2012): Toxicology and overdose of atypical antipsychotic. J Emerg Med. 43 (5):906-13.

Oguche S, Bukbuk D and Watila I (2007): Pattern of hospital admissions of children with poisoning in the Sudano- Sahelian North eastern Nigeria. Niger J Clin Pract. 10 (2):111-5.

Olson K (2012): Emergency evaluation and treatment. In Olson's poisoning and drug overdose Olson $\mathrm{K}$ R et al., (eds), $6^{\text {th }}$ ed., San Francisco, California. Ch I. pp.1-68.

Pacher S and Kecskemeti V (2004): Cardiovascular side effects of new antidepressants and antipsychotics: New drugs, old concerns? Curr Pharm. 10 (20):2463-2475.

Persson H, Sjoberg G, Haines J et al., (1998): Poisoning severity score. Grading of acute poisoning. J Toxicol Clin Toxicol. 36: 205-13.
Pratt D and Kaplan M (2000): Evaluation of abnormal liver-enzyme results in a symptomatic patients. New Engl J Med. 342:1266-71.

Saltik S and Basgul S (2012): Neuroleptic malignant syndrome due to risperidone overdose in a child: A Case Report Bulletin of Clinical Psychopharmacology. 22 (1):44-48.

Seitz D and Gill S (2009): Neuroleptic malignant syndrome complicating antipsychotic treatment of delirium or agitation in medical and surgical patients: Case reports and a review of the literature. Psychosomatics. 50 (1):8-15.

Shirzadi A and Ghaemi S (2006): Side effects of atypical antipsychotics: Extrapyramidal symptoms and the metabolic syndrome. Harv Rev Psychiatry. 14(3):152-64.

Shy K and Rund D (2011): Psychotropic medications. In Tintinalli JE, Ruiz E, Pharmacol. 11: 59-67.

Soueif M (1994): Extent and patterns of drug use among students and working class men in Egypt. Cairo: National Centre for Social and Criminological Research.

Steven B (2006): General considerations in the evaluation and treatment of poisoning. In: Book manual of overdose and poisoning. Linden $\mathrm{C} \mathrm{H}$ et al., (eds), $1^{\text {st }}$ ed., Lippincott Williams and Wilkins. Ch 1. pp: 1-7.

Strawn J, Keck P and Caroff S (2007): Neuroleptic malignant syndrome. Am J Psychiatry. 164 (6):870-6.

Trenton A, Currier G and Zwemer F (2003): Fatalities associated with therapeutic use and overdose of atypical anti psychotics. CNS Drugs. 17:307-24.

Vernon D and Gleich M (1997): Poisoning and drug overdose. Critical Care Clinics. 13 (3): 647667.

Wills B and Erickson T (2005): Drug and toxin associated seizures. Med Clin North Am. 89: 1297-321.

Yasan A, Danis R, Tamam L et al., (2008): Sociocultural features and sex profile of the individuals with serious suicide attempts in southeastern Turkey: A one-year survey. Suicide Life Threat Behav. 38: 467-80.

Zakaria Z, Shervin T, Mohammad T et al., (2012): Bilateral dislocation of temporo mandibular joint induced by Haloperidol following suicide attempt: A Case Repor Acta Medica Iranica. $50(3): 1-7$.

Zareba W and Lin D (2003): Antipsychotic drugs and QT interval prolongation. Biol Psychiatr. 74: 291-306. 


\section{الملخص العربي \\ دراسة مستقبلية لمقياس خطورة السمية في حالات التسمم بمضادات الذهان التي ادخلت مركز علاج السموم

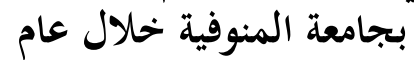

سامي عبد الهادي حماد و نيرة فهمي جرجس وصفاء عبد الظاهر أمين و عزة وجيه زناتي و هايدي مصطفى ابو حطب1 المقدمة: تصنف مضادات الذهان من أكثر خمس مواد يتعرض لها الإنسان. من الشائع زيادة جرعة أدوية مضادات الذهان.

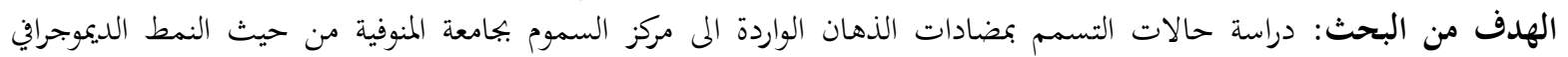

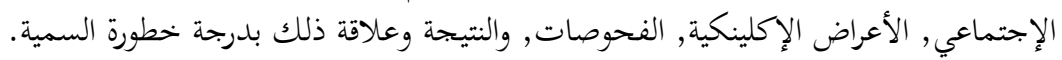

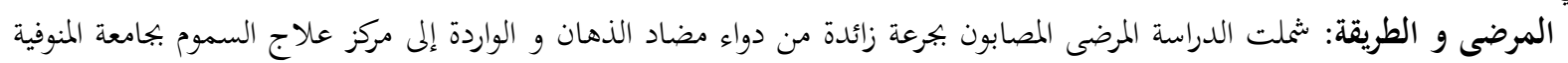

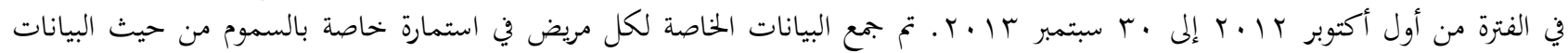

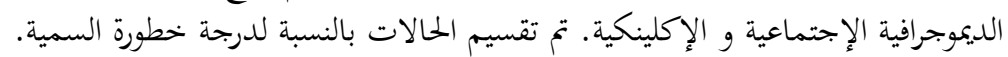

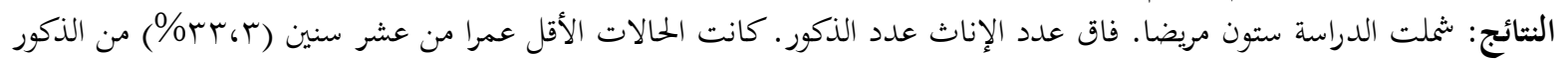

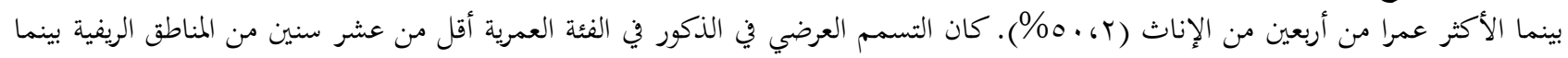

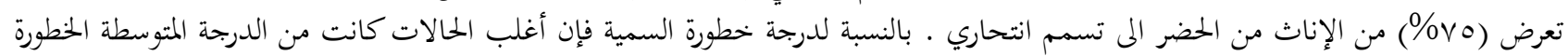

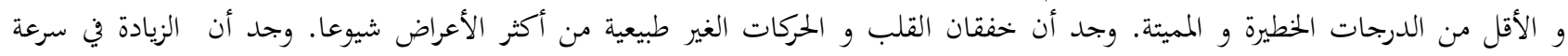

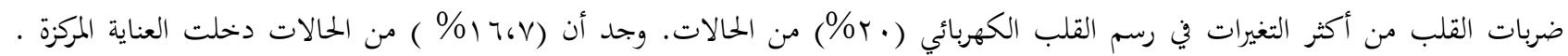

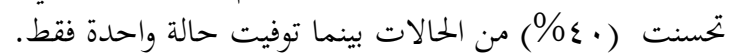

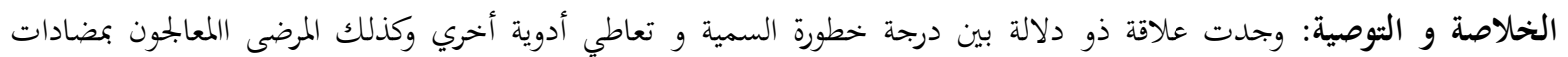

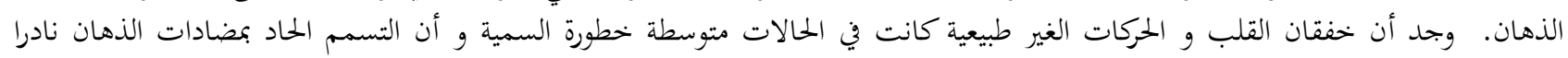

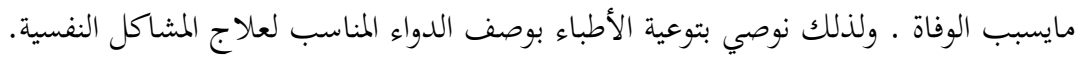

